\title{
Don't lose sleep over neurodegeneration-it helps clear amyloid beta
}

\author{
John McKinley ${ }^{1,2}{ }^{*}$, Allan McCarthy ${ }^{1}$ and Timothy Lynch ${ }^{1}$ \\ ${ }^{1}$ Department of Neurology, The Dublin Neurological Institute at The Mater Misericordiae University Hospital, Dublin, Ireland \\ ${ }^{2}$ Dublin Academic Medical Centre, Dublin Neurological Institute, Dublin, Ireland \\ *Correspondence: johnmckinley@doctors.org.uk
}

Edited by:

Alberto J. Espay, University of Cincinnati, USA

Reviewed by:

Ron Postuma, Montreal General Hospital, Canada

Jennifer Molano, University of Cincinnati Medical Center, USA

Keywords: sleep, amyloid, neurodegeneration, cerebrospinal fluid

\section{A commentary on}

Sleep drives metabolite clearance from the adult brain

by Xie L, Kang H, Xu Q, Chen MJ, Liao Y, Thiyagarajan M, O'Donnell J, Christensen DJ, Nicholson C, Iliff JJ, Takano T, Deane R, Nedergaard M. Science (2013) 342:373-77. doi: 10.1126/science.1241224

Xie et al. in their article entitled "sleep drives metabolite clearance from the adult brain" in Science (342: 373-377) (1), provide an important insight into the potential physiology behind the restorative and homeostatic nature of sleep, a basic requirement for normal brain function. This study, utilizing a mouse model explores the clearance of peptides such as amyloid beta, which is implicated in the pathogenesis of Alzheimer's disease (2) and may offer a platform for the investigation of other forms of neurodegeneration associated with other putative neurotoxic proteins.

Key to understanding their paradigm is an exploration of the differences between physiological clearance of cellular waste from the central nervous system and peripheral tissues. Systemic waste interstitial proteins are transported via lymph vessels and the systemic circulation to the liver for degradation (1). The brain lacks this mechanism and relies on the recirculation of cerebrospinal fluid, which acquires waste proteins as it flows through the interstitial spaces surrounding brain cells (2) before interfacing with the systemic circulation at the arachnoid granulations. This convective mechanism constitutes the glymphatic system and relies on aquaporin 4 (AQP4) water channels for effective clearance of waste solute. The authors highlight that many of the putative proteins associated with neurodegeneration are found in the interstitial fluid surrounding brain cells and that knocking out AQP4 channels for example reduces the clearance of amyloid beta $(\mathrm{A} \beta)$ by $65 \%$ (1).

Xie et al. used a mouse model, with an implanted cannula for delivery of a fluorescent tracer via the cisterna magna into the CSF, to observe real-time movement of CSF in vivo using two-photon imaging (1). This technique allowed the influx of CSF into the murine cerebral cortex to be monitored "real-time" in three states; sleep, awake, and anesthetized.

Electrocorticography (ECoG) and electromyography (EMG) determined sleepwake status, with a sleep state practically defined as an increased power of slow waves and an awake state associated with a relative reduction in slow waves and increase in power of fast waves (1).

On entering sleep there was a "robust influx" of fluorescent CSF tracer along the periarterial spaces, subpial region, and into the brain parenchyma (1). Sleep was confirmed by the presence of a high power of slow waves on the ECoG.

On awakening, CSF tracer influx reduced dramatically by $95 \%$ (1). The state of wakefulness was confirmed by a change in the ECoG (1).

These results were replicated in a cohort of mice, examined firstly whilst awake. The intracisternal infusion of fluorescent CSF tracer was associated with only minimal influx into the brain. Then on induction of anesthesia there was a rapid influx of CSF tracer along the periarterial spaces into the brain parenchyma, similar to naturally sleeping mice (1).

They postulate that CSF influx into brain parenchyma is not simply a function of arterial pulsation and blood pressure but rather the interstitial space volume is dynamic and contracts during wakefulness and expands during sleep (1).

To test this, they used the real-time iontophoretic tetramethylammonium $\left(\mathrm{TMA}^{+}\right)$method whereby $\mathrm{TMA}^{+}$was delivered via an iontophoresis microelectrode in the vicinity of a $\mathrm{TMA}^{+}$-sensitive microelectrode stationed approximately $150 \mu \mathrm{m}$ away in the interstitial space. In the paradigm a small interstitial space results in reduced $\mathrm{TMA}^{+}$dilution (as volume of interstitial space is reduced) after delivery and thus higher levels of residual $\mathrm{TMA}^{+}$ are detected by the sensing electrode (1) and vice versa. Essentially they confirmed that the interstitial space volume fraction was increased by over $60 \%$ in anesthetized or sleeping mice compared to when they were awake (1).

Knowing that approximately $65 \%$ of exogenously delivered $A \beta$ is cleared by the glymphatic system they decided to explore whether interstitial $A \beta$ is cleared most efficiently during sleep (1). Radiolabeled ${ }^{125} \mathrm{I}-\mathrm{A} \beta_{1-40}$ was injected into the cortex of awake mice, sleeping mice, and anesthetized mice (1). Brains were examined for levels of residual ${ }^{125} \mathrm{I}-\mathrm{A} \beta_{1-40}$ between 10 and $240 \mathrm{~min}$ after injection and they established that $A \beta$ was cleared twice as fast in sleeping and anesthetized mice compared to awake mice. Furthermore, to 
Table 1 | Summary of four principal experimental methodologies, results, and implications.

\begin{tabular}{|c|c|c|c|}
\hline Experiment & Methods & Results & Implications \\
\hline $\begin{array}{l}\text { Real-time observation of } \\
\text { CSF influx to cerebral } \\
\text { cortex }\end{array}$ & $\begin{array}{l}\text { Infusion of fluoroscopic CSF tracer via a } \\
\text { cannula implanted into the cisterna magna. } \\
\text { Imaging in vivo using two-photon imaging } \\
\text { in three states of arousal (awake, asleep, } \\
\text { and under anesthesia) }\end{array}$ & $\begin{array}{l}\text { When asleep: "robust influx" of } \\
\text { fluorescent CSF tracer along the } \\
\text { periarterial spaces, subpial } \\
\text { regions, and parenchyma } \\
\text { When awake: CSF influx reduced } \\
\text { "dramatically" by } 95 \% \\
\text { When anesthetized: on induction } \\
\text { there was a rapid influx of CSF } \\
\text { similar to the sleeping state }\end{array}$ & $\begin{array}{l}\text { Interstitial space volume is dynamic } \\
\text { and a function of the state of arousal } \\
\text { whether that be physiological states } \\
\text { such as sleep or wakefulness or } \\
\text { artificially induced states such as } \\
\text { anesthesia }\end{array}$ \\
\hline
\end{tabular}

\author{
Assessment of interstitial \\ space volume during \\ different states of arousal \\ (sleep, wakefulness, or \\ anesthesia)
}

\section{Assessment of $A \beta$} clearance during different states of arousal (sleep, wakefulness, or anesthesia)

Determining if interstitial space volume is influenced by arousal state or circadian rhythm
Iontophoretic Tetramethylammonium method $\left(\mathrm{TMA}^{+}\right)$: $\mathrm{TMA}^{+}$delivered to cortex via an iontophoresis microelectrode situated $150 \mu \mathrm{m}$ from a $\mathrm{TMA}^{+}$sensitive microelectrode located in the interstitial space

\author{
Radiolabeled ${ }^{125} \mid-A \beta_{1-40}$ injected into the \\ cerebral cortex. Brains harvested between \\ 10 and 240 min after injection and residual \\ ${ }^{125} \mathrm{I}-\mathrm{A} \beta_{1-40}$ measured
}

\begin{abstract}
Based on the premise that wakefulness is driven by noradrenergic tone, glymphatic tracer influx was assessed (after the local administration of adrenergic antagonist) using the fluoroscopic tracer method outlined above. Furthermore, interstitial space volume was assessed in response to noradrenergic antagonist using the iontophoretic $\mathrm{TMA}^{+}$method outlined above
\end{abstract}

\section{Interstitial space volume fraction increased by over $60 \%$ during the sleeping or anesthetized state}

\section{$A \beta$ cleared twofold faster in sleeping/anesthetized mice compared to awake mice}

Confirms that interstitial space volume is dynamic and influenced by state of arousal

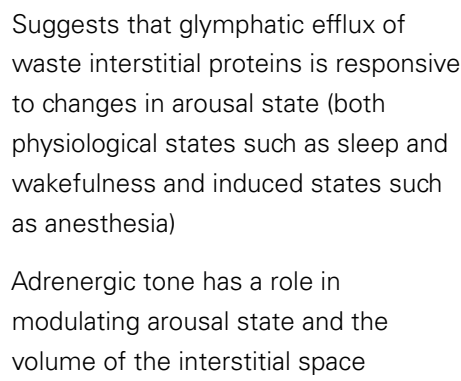
wakefulness and induced states such as anesthesia)

Adrenergic tone has a role in modulating arousal state and the volume of the interstitial space

an increase in CSF tracer influx

"more comparable" to that seen

in the sleeping/anesthetized

state than that seen during

wakefulness. Adrenergic

antagonist resulted in significant

increase in interstitial space

volume fraction account for additional receptor-mediated clearance of $A \beta$ across the blood brain barrier, an inert ${ }^{14} \mathrm{C}$-inulin tracer was shown to be cleared more than twice as fast in sleeping and anesthetized mice compared to awake mice (1).

There are two possible contributory factors. Either interstitial space volume is influenced by circadian rhythm or it is a function of the sleep-wake state of the individual (1). The authors assert that it is sleep-wake state rather than circadian rhythm that is fundamental as induction of anesthesia at a time usually associated with natural wakefulness results in identical interstitial space volume and $A \beta$ clearance to that found in natural sleep (1). Based on this premise, they postulate that the noradrenergic (NA) system controls the interstitial space volume by driving arousal. Thus, they hypothesized that blockade of NA during wakefulness would result in an increase in CSF influx into the brain. Xie et al. infused an adrenergic antagonist into the cisterna magna during wakefulness whilst measuring CSF influx using their original fluorescent CSF tracer method and found that the infusion of adrenergic antagonists increased CSF influx similar to that seen in the sleeping state (1). Using the $\mathrm{TMA}^{+}$iontophoresis method they further confirmed that NA antagonism increased the brain interstitial volume in a similar fashion to sleep and anesthesia (1). The four principal experiments by Xie et al. (summarized in Table 1) have elucidated how cortical CSF influx and interstitial space volume are influenced by arousal state and how pharmacological intervention in the form of anesthesia or adrenergic antagonism can modify these parameters.

Why do we need to sleep? The authors postulate that sleep is the homeostatic method responsible for the clearance of toxic metabolic byproducts accumulated during wakefulness. This study by Xie et al. raises many questions in the study of neurodegeneration. Could NA antagonists impact on the accumulation of putative toxic proteins in Alzheimer's disease and other forms of neurodegeneration? Is rapid eye movement (REM) sleep disorder merely a manifestation of alpha synucleinopathies or could it be implicated in pathogenesis? Why do some patients with Parkinson's disease derive sleep benefit? What causes fluctuating arousal in dementia with Lewy bodies (DLB)? Does sleep-wake state influence volumetric 
analysis in MRI? Given the invasive nature of the experimental methodologies used they could not be reproduced in humans in their current format, however they could provide a platform for the investigation of clearance of other neurotoxic proteins involved in neurodegeneration, using a Parkinson's disease mouse model for example. Furthermore, they may be useful for investigation of potential drugs to promote the clearance of neurotoxic proteins, whether they be currently used adrenergic antagonist drugs or novel agents. When we try to encourage a good nights sleep for our patients perhaps we are doing them more good than we thought.

\section{ACKNOWLEDGMENTS}

Financial Disclosures: Dr. McKinley and Dr. McCarthy report no disclosures. Professor
Lynch receives honoraria from Abbot, Boehringer Ingelheim, Lundbeck, and Orion. He has received educational grants from Bayler Schering, Biogen Idec, Lundbeck, and Medtronic. He has received grants from the Irish Institute of Clinical Neuroscience, the Mater College and PRTL1 funding. Professor Lynch sits on the advisory boards of Abbot, Novartis, UCB Pharma, Teva, Merck Serono, and Biogen Idec. Research Funding: N/A. Authorship roles: Dr. McKinley drafted the manuscript and it was reviewed and revised by Dr. McCarthy and Professor Lynch.

\section{REFERENCES}

1. Xie L, Kang H, Xu Q, Chen MJ, Liao Y, Thiyagarajan $\mathrm{M}$, et al. Sleep drives metabolite clearance from the adult brain. Science (2013) 342:373-7. doi:10.1126/science.1241224

2. Herculano-Houzel S. Sleep it out. Science (2013) 342:316-7. doi:10.1126/science.1245798

Received: 13 November 2013; accepted: 07 December 2013; published online: 19 December 2013.

Citation: McKinley J, McCarthy A and Lynch T (2013) Don't lose sleep over neurodegeneration-it helps clear amyloid beta. Front. Neurol. 4:206. doi: 10.3389/fneur.2013.00206

This article was submitted to Movement Disorders, a section of the journal Frontiers in Neurology.

Copyright $\odot 2013$ McKinley, McCarthy and Lynch. This is an open-access article distributed under the terms of the Creative Commons Attribution License (CC BY). The use, distribution or reproduction in other forums is permitted, provided the original author(s) or licensor are credited and that the original publication in this journal is cited, in accordance with accepted academic practice. No use, distribution or reproduction is permitted which does not comply with these terms. 